\title{
Répercussions d'une alimentation azotée ammoniacale sur le métabolisme carboné photosynthétique chez le soja (Glycine max L Merr)
}

\author{
P M Kpodar, JC Latché*, G Cavalié \\ Université Paul Sabatier, centre de physiologie végétale, 118, route de Narbonne, 31062 Toulouse Cedex, France
}

(Reçu le 20 septembre 1991; accepté le 16 janvier 1992)

\begin{abstract}
Résumé - Comparativement à une alimentation nitrique, une alimentation azotée ammoniacale entraîne des perturbations dans la croissance du soja. La masse de matière sèche par plante est réduite, de même que la surface des feuilles dont les limbes présentent successivement des symptômes de flétrissement, de chlorose et enfin de nécrose. L'assimilation carbonée photosynthétique a été suivie après fixation de ${ }^{14} \mathrm{CO}_{2}$ à divers stades de développement. Les végétaux cultivés sur nitrate se caractérisent par une incorporation supérieure de radiocarbone dans les acides organiques, essentiellement l'acide malique. Dans les plantes alimentées en azote ammoniacal, on observe un marquage plus élevé du saccharose et surtout des acides aminés et amides libres. Parmi ces derniers, l'importance de la sérine suggère que cet amino-acide pourrait participer à la détoxification des cellules vis-à-vis des ions ammonium. La photosynthèse nette est inférieure en nutrition ammoniacale. Ce résultat est à relier à une augmentation de la résistance stomatique, à une diminution des concentrations en chlorophylles et en protéines foliaires et à une baisse de l'activité carboxylasique de la Rubisco. En outre, les teneurs en Rubisco, dosées par immunoélectrophorèse, sont plus faibles. L'ensemble de ces données explique l'effet néfaste des ions ammonium et illustre quelques interactions entre nutrition azotée et nutrition carbonée.
\end{abstract}

soja = Glycine $\max /$ ammonium / métabolisme photosynthétique / Rubisco / sérine

Summary - Effects of ammonium nitrogen nutrition on soybean (Glycine max L Merr) photosynthetic carbon metabolism. Ammonium ions compared to nitrate ions as the source of nitrogen decreased dry matter production and reduced size and leaf area of soybean plants. Leaves wilted progressively and the margins became chlorotic and partially necrotic when old. Photosynthetic carbon assimilation was investigated by following ${ }^{14} \mathrm{CO}_{2}$ incorporation. Nitrate increased ${ }^{14} \mathrm{C}$ incorporation in the anionic fraction; malic acid was the most labelled compound. In the plants grown with ammonium, labelling of sucrose and especially of free amino acids was higher. The accumulation of serine shows that this amino acid participates in ammonia detoxification. Net photosynthetic rate was lower in plants grown with ammonium. This can be explained by an increase in stomatal resistance, a decrease in chlorophyll and in leaf soluble protein content, and variations in ribulose bisphosphate carboxylase activity. Moreover, the amount of Rubisco protein, determined by rocket immunoelectrophoresis, decreased with ammonium feeding. These data explain the toxic effect of ammonium ions and illustrate the interactions between nitrogen and carbon nutrition in soybean plants.

soybean = Glycine $\max /$ ammonium $/$ photosynthetic metabolism $/$ Rubisco $/$ serine

* Correspondance et tirés à part 


\section{INTRODUCTION}

Une alimentation azotée ammoniacale entraîne souvent un net ralentissement de la croissance des plantes supérieures (Kirkby et Mengel, 1967; Kpodar et al, 1978; Bernardo et al, 1984; Chaillou et al, 1986; Shelp, 1987). De façon générale, ces perturbations sont toutefois moins marquées chez les Graminées que chez les Dicotylédones (Salsac et al, 1987).

L'effet toxique des ions ammonium est encore mal expliqué et il paraît être la résultante de divers phénomènes. On peut d'abord citer l'acidification du milieu de culture consécutive à l'absorption et à l'utilisation racinaire du $\mathrm{NH}_{4}^{+}$, le déficit en cations minéraux et les faibles teneurs en acides organiques souvent constatés chez les végétaux soumis à une nutrition ammoniacale, leur richesse parfois excessive en anions minéraux, des modifications dans la compartimentation cellulaire du phosphate inorganique (Salsac et al, 1987).

Le métabolisme carboné photosynthétique semble également affecté. À forte concentration, les ions ammonium sont des découplants des photophosphorylations (De Benedetti et al, 1976; Archhireddy et al, 1983) et l'apport d'azote sous forme de $\mathrm{NH}_{4}^{+}$provoque, outre la diminution des teneurs en chlorophylles (Bernardo et al, 1984; Magalhaes et Wilcox, 1984; Mac Leod et Ormrod, 1985), une baisse sensible de la fixation du $\mathrm{CO}_{2}$; elle a été signalée notamment chez le haricot (Marques et al, 1983), le blé (Walker et al, 1984) et Arabidopsis thaliana (Grumbles, 1987).

Chez le blé cependant, aucune variation de l'intensité photosynthétique en réponse à une alimentation ammoniacale n'a été décrite par Sharma et Sirohi (1987) et une constatation similaire a été effectuée à partir de cellules foliaires isolées de cotonnier (Rehfeld et Jensen, 1973) et chez le maïs (Grumbles, 1987). La présence d'ions $\mathrm{NH}_{4}^{+}$dans le milieu de culture conduit même à une augmentation de la photosynthèse nette de cellules d'épinard (Woo et Canvin, 1980), à relier éventuellement à un accroissement de l'activité carboxylasique de la Rubisco (De Benedetti et al, 1976; Hall et al, 1984). Enfin le devenir du carbone fixé peut être modifié, la biosynthèse d'amidon étant par exemple perturbée chez le concombre (Wakiuchi et al, 1971).
L'ensemble de ces données montre que, d'une part, les résultats acquis sont parfois contradictoires et que, d'autre part, ils ont souvent été obtenus de façon fragmentaire à partir de végétaux variés. Ceci nous a incités à entreprendre sur un même matériel, le soja, une étude détaillée des répercussions d'une nutrition azotée ammoniacale sur la photosynthèse. L'importance de l'assimilation du $\mathrm{CO}_{2}$ et la répartition du carbone fixé dans les différents métabolites ont été d'abord précisées; l'évolution de divers paramètres photosynthétiques a été ensuite suivie comparativement chez des plantes cultivées en présence d'azote nitrique ou ammoniacal: résistance stomatique, richesse en chlorophylles, teneurs en protéines solubles foliaires et en Rubisco, activité carboxylasique et degré d'activation de cette dernière enzyme.

\section{MATÉRIEL ET MÉTHODES}

\section{Matériel végétal}

Le soja, Glycine max (L) Merr, variété M13, à développement de type déterminé, est cultivé en conditions contrôlées (héméropériode, 14 h; éclairement, $400 \mu \mathrm{mol}$ de photons $\cdot \mathrm{m}^{-2} \cdot \mathrm{s}^{-1}$; température, $25^{\circ} \mathrm{C}$ en phase éclairée, $18^{\circ} \mathrm{C}$ en phase obscure; humidité relative, $60-80 \%$ ), sur sable imbibé de solutions nutritives synthétiques. Les 2 solutions utilisées (Kpodar et al, 1978) renferment des concentrations identiques de $\mathrm{N}, \mathrm{K}, \mathrm{Ca}$ et $\mathrm{Mg}$ mais l'azote est apporté soit sous forme de nitrate $\left(\mathrm{Ca}\left(\mathrm{NO}_{3}\right)_{2} 2,5 \cdot 10^{-3} \mathrm{~mol} \cdot \mathrm{I}^{-1}\right.$ et $\left.\mathrm{KNO}_{3} 5 \cdot 10^{-3} \mathrm{~mol} \cdot \mathrm{I}^{-1}\right)$, soit sous forme d'ammonium $\left(\mathrm{NH}_{4} \mathrm{H}_{2} \mathrm{PO}_{4}\right.$ $2 \cdot 10^{-3} \mathrm{~mol} \cdot \mathrm{I}^{-1}, \quad\left(\mathrm{NH}_{4}\right)_{2} \mathrm{SO}_{4} 1 \cdot 10^{-3} \mathrm{~mol}^{-1}$ et $\mathrm{NH}_{4} \mathrm{Cl}$ $\left.\left.6 \cdot 10^{-3} \mathrm{~mol} \cdot\right|^{-1}\right)$. En cours de culture, elles sont renouvelées quotidiennement, ou tous les $2 \mathrm{j}$, afin de minimiser les modifications de $\mathrm{pH}$ liées au développement des plantes: l'utilisation des ions $\mathrm{NO}_{\overline{3}}$ entraine en effet une alcalinisation de la solution, le phénomène inverse étant observé en nutrition ammoniacale.

Les végétaux sont d'abord cultivés pendant 14 j sur milieu nitrate; un lot de plantes est ensuite transféré sur milieu ammonium, alors que le second lot est maintenu sur le milieu d'origine. $\mathrm{Ce}$ protocole permet par comparaison de déterminer l'ordre chronologique d'apparition des effets des ions $\mathrm{NH}_{4}^{+}$et d'en préciser l'évolution.

Dans tous les cas, l'expérimentation porte sur la $3^{e}$ feuille trifoliolée récoltée, sauf indication contraire, environ $3 \mathrm{~h}$ après le début de l'héméropériode; elle correspond à la feuille la plus récemment différenciée ayant atteint, au moment du 
changement de milieu, $50-60 \%$ de sa surface définitive.

\section{Assimilation de ${ }^{14} \mathrm{CO}_{2}$, identification des métabolites marqués et mesures de radioactivité}

Les expériences de fixation de ${ }^{14} \mathrm{CO}_{2}$ ont été réalisées à partir de lots de 12 disques de limbe foliaire (diamètre, $24 \mathrm{~mm}$ ), prélevés sur 6-10 feuilles. Les disques sont disposés dans des boîtes de $\mathrm{Pe}$ tri renfermant quelques $\mathrm{ml}$ de milieu de culture dilué au demi (milieu nitrate ou ammonium selon l'échantillon), ce qui permet d'éviter la déshydratation des tissus. Le matériel végétal est alors introduit dans une enceinte de verre soumise à un éclairement de $320 \mu \mathrm{mol}$ de photons $\mathrm{m}^{-2} \cdot \mathrm{s}^{-1}$, la température étant comprise entre 20 et $22^{\circ} \mathrm{C}$. Après balayage de l'enceinte ( $15 \mathrm{~min})$ par un courant d'air dépourvu de $\mathrm{CO}_{2}$, ayant pour but de favoriser l'ouverture des stomates, l'addition d'acide lactique à un mélange de bicarbonate de sodium inerte et de $\mathrm{NaH}^{14} \mathrm{CO}_{3}$ (radioactivité spécifique, $2,05 \mathrm{GBq} \mathrm{mmol}^{-1}, \mathrm{CEA}$ ) provoque un dégagement de gaz carbonique (concentration, $400 \mathrm{vpm}$; radioactivité par expérience, $370 \mathrm{kBq}$ ). La durée de l'assimilation photosynthétique a été fixée à $5 \mathrm{~min}$, des essais préliminaires ayant montré une proportionalité entre fixation et durée de l'expérience pour des temps de photosynthèse n'excédant pas $10 \mathrm{~min}$. Le matériel végétal est ensuite fixé dans de l'éthanol à $96^{\circ} \mathrm{GL}$ refroidi à $-20^{\circ} \mathrm{C}$, puis broyé

Le protocole retenu pour l'extraction à froid (mélange eau-glace) des métabolites solubles en $\mathrm{mi}$ lieux hydroéthanoliques, et leur séparation par chromatographie sur échangeurs d'ions, puis par chromatographie de partage, est identique à celui décrit dans une publication antérieure (Kpodar et al, 1978). Après autoradiographie (films Kodak X Omat $S$ ), le marquage des composés est déterminé par scintillation en milieu liquide à l'aide d'un spectromètre Packard, type CSL $460 \mathrm{C}$. La radioactivité du culot d'extraction insoluble, préalablement séché sous vide en présence de $\mathrm{CaCl}_{2}$ et broyé, est mesurée selon la même technique, après combustion (oxidizer Packard, type Tricarb 306).

\section{Dosage des constituants azotés et glucidiques}

Les analyses sont effectuées à partir de tissus foliaires lyophilisés et réduits en poudre.

Les teneurs en acides aminés et amides libres, extraits à froid dans l'acide trichloracétique selon Guitton (1964), sont déterminées au moyen d'un analyseur d'acides aminés Beckman, type $119 \mathrm{CL}$.
L'extraction des constituants glucidiques simples est réalisée, à $80^{\circ} \mathrm{C}$, dans l'éthanol à $80^{\circ} \mathrm{GL}$ ( 3 épuisements successifs). Après élimination de l'alcool par évaporation sous pression réduite, l'extrait brut est passé successivement sur colonne d'échangeurs de cations, puis d'anions. Les sucres, recueillis dans l'effluent, sont dosés par l'anthrone (méthode de Dreywood modifiée par Shields et Burnett, 1960).

L'amidon est solubilisé, à partir du culot d'extraction éthanolique, par chauffage à $100^{\circ} \mathrm{C}$, en présence de potasse $0,2 \mathrm{~N}$, pendant 30 min (Rufty et Huber, 1983). Après refroidissement, le $\mathrm{pH}$ est amené à 4,5 par addition d'acide acétique $\mathrm{N}$ et on procède à une hydrolyse de l'amidon (tampon acétate de sodium $50 \mathrm{mmol}^{-1} \mathrm{f}^{-1} \mathrm{pH} 4,5,50^{\circ} \mathrm{C}, 20 \mathrm{~min}$ ) par une $\alpha$-amyloglucosidase du commerce (EC 3.2.1.3, Sigma, 35 unités $\cdot \mathrm{ml}^{-1}$ ). Le glucose libéré est ensuite dosé par l'anthrone.

\section{Mesure des teneurs en chlorophylles}

Les pigments sont extraits par broyage des tissus frais dans un mélange d'acétone $(80 \%)$ et d'eau (20\%), selon Arnon (1949). Après mesure à $652 \mathrm{~nm}$ de la densité optique de la solution obtenue, la concentration totale en chlorophylles est calculée en utilisant le coefficient de Bruinsma (1961).

\section{Détermination de la résistance stomatique}

Elle a été effectuée à l'aide d'un poromètre à diffusion de vapeur d'eau (type MK II Delta T Devices), d'après la technique décrite par Cruiziat et al (1979).

\section{Extraction, mesure de l'activité carboxylasique et détermination des teneurs en ribulose bisphosphate carboxylase/oxygénase (Rubisco, EC 4.1.1.39)}

Le protocole d'extraction décrit par Ranty et Cavalié (1982) a été retenu. L'échantillon végétal, constitué par $5 \mathrm{~g}$ de limbe. frais dénervuré, est broyé dans $20 \mathrm{ml}$ de tampon Tris- $\mathrm{HCl} 0,1 \mathrm{~mol} \cdot \mathrm{l}^{-1}$ $\mathrm{pH} 7,5$, renfermant du polyclar et du polyéthylène glycol 6000 (respectivement 0,1 et $0,02 \mathrm{~g}$ par $\mathrm{g}$ de végétal), du dithiothréitol $\left(10 \mathrm{mmol} \cdot \mathrm{I}^{-1}\right)$ et de l'acide éthylène diamine-tétraacétique $\left(1 \mathrm{mmol} \cdot \mathrm{I}^{-1}\right)$. Le broyat est centrifugé $\left(25000 \mathrm{~g}, 15 \mathrm{~min}, 2^{\circ} \mathrm{C}\right)$ et le surnageant, filtré sur Miracloth, constitue l'extrait enzymatique dont l'activité carboxylasique est mesurée par méthode radiochimique (Raghavendra et 
Das, 1977). On procède d'abord à une activation du biocatalyseur $\left(5 \mathrm{~min}, 30^{\circ} \mathrm{C}\right.$, tampon Tris- $\mathrm{HCl}$ $50 \mathrm{mmol} \cdot \mathrm{I}^{-1}$, pH 8 ) en présence de $\mathrm{MgCl}_{2}$ $\left(10 \mathrm{mmol} \cdot \mathrm{I}^{-1}\right)$ et de $\mathrm{KH}^{14} \mathrm{CO}_{3}\left(20 \mathrm{mmol} \cdot \mathrm{I}^{-1}\right)$; l'apport de ribulose 1,5 bisphosphate $\left(0,5 \mathrm{mmol} \cdot \mathrm{I}^{-1}\right)$ déclenche la réaction, stoppée au bout de $3 \mathrm{~min}$ par addition d'HCl $5 \mathrm{~N}$. La radioactivité incorporée dans la fraction acide stable, que l'on mesure sur une partie aliquote du mélange réactionnel préalablement évaporée à sec dans une étuve ventilée, correspond à l'activité totale ou potentielle. Parallèlement, l'addition de l'extrait enzymatique, aussitôt après l'extraction et sans phase d'activation préalable, dans un milieu renfermant les substrats (ribulose 1,5 bisphosphate et bicarbonate) et le $\mathrm{MgCl}_{2}$, conduit à l'estimation de l'activité initiale (Torisky et Servaites, 1984). Ces 2 valeurs permettent de calculer le degré d'activation A du biocatalyseur, donné par la relation:

$$
A(\%)=\frac{\text { activité initiale }}{\text { activité totale }} \times 100 .
$$

Les teneurs en Rubisco ont été déterminées par immunoélectrophorèse (Laurell, 1966), l'immunosérum spécifique de la Rubisco du soja ayant été obtenu au laboratoire (Grima-Pettenati, 1985). Les conditions expérimentales d'immunoélectrophorèse ont été définies par Courtiade (1983).

\section{Dosage des protéines}

II est réalisé par la méthode de Lowry et al (1951) modifiée par Bensadoun et Weinstein (1976).

\section{RÉSULTATS}

\section{Incidence de l'alimentation ammoniacale sur la croissance et le développement de la plante}

La masse de matière sèche par plante est inférieure en alimentation ammoniacale, le phénomène s'accentuant en fonction de la durée de la culture. Ainsi, les diminutions observées par rapport aux échantillons cultivés sur nitrate sont en moyenne de $14 \%, 33 \%$ et $39 \%$ après respectivement 10,20 et $25 \mathrm{j}$ de transfert des végétaux sur milieu ammonium. Les plantes sont de taille plus réduite, la surface des feuilles est plus faible et le limbe, dont la masse spécifique augmente $(+15-20 \%)$, présente des symptômes de flétrissement, de chlorose et enfin de nécrose dans les organes âgés.

Cependant le développement du végétal paraît beaucoup moins perturbé: le nombre total de feuilles est sensiblement identique quelle que soit la composition du milieu nutritif, de même que les proportions pondérales des différents organes végétatifs.

\section{Assimilation photosynthétique du ${ }^{14} \mathrm{CO}_{2}$}

\section{Photosynthèse nette}

Les taux de photosynthèse nette, calculés à partir de l'assimilation de ${ }^{14} \mathrm{CO}_{2}$ par des disques de limbe foliaire, sont consignés dans le tableau I. La photosynthèse du soja, plante de type $\mathrm{C}_{3}$, correspond à la fixation horaire moyenne de 20-25 mg d'anhydride carbonique par $\mathrm{dm}^{2}$ de surface foliaire (Mondal et Brenner, 1978). Dans le cas des plantes "nitrate", la feuille en fin de croissance ou adulte (20, $25^{\mathrm{e}} \mathrm{j}$ de culture), conduit à des valeurs similaires. Les diminutions observées ensuite à partir des échantillons plus âgés, conformes aux résultats obtenus notamment par Latché et al (1986), proviennent non seulement d'une baisse de l'intensité de fixation du $\mathrm{CO}_{2}$, mais aussi d'une accélération du métabolisme photorespiratoire (Secor et al, 1982); Latché et al, 1983).

L'apport d'azote sous forme ammoniacale durant $5 \mathrm{j}$ (du $15^{\mathrm{e}}$ au $20^{\mathrm{e}}$ j de culture) ne modifie pas la photosynthèse nette de façon significative. Après une baisse d'environ 35\% entre le $20^{\mathrm{e}}$ et le $25^{\mathrm{e}} \mathrm{j}$, la quantité de $\mathrm{CO}_{2}$ assimilé se maintient constante en valeur $a b-$ solue jusqu'au $30^{\mathrm{e}} \mathrm{j}$, puis elle chute à nouveau au dernier stade analysé $(-28 \%)$. Ces résultats, joints à l'existence d'une période de la-

Tableau 1. Photosynthèse nette, en $\mathrm{mg}$ de $\mathrm{CO}_{2}$ fixé par $\mathrm{dm}^{2}$ de surface foliaire et par h, en fonction de la composition du milieu nutritif. Moyenne de 4 échantillons.

\begin{tabular}{lcccc}
\hline \multicolumn{1}{c}{ Jours après semis } & 20 & 25 & 30 & 35 \\
\hline Plantes «nitrate» & $18,8 \pm 1,3$ & $19,4 \pm 1,2$ & $14,0 \pm 1,1$ & $12,0 \pm 1,1$ \\
Plantes «ammonium» & $18,4 \pm 1,4$ & $12,6 \pm 1,2$ & $12,2 \pm 0,9$ & $8,6 \pm 0,9$ \\
\hline
\end{tabular}


tence indispensable pour que l'effet des ions $\mathrm{NH}_{4}^{+}$se manifeste, pourraient être le signe de possibilités d'adaptation temporaire du végétal à une forme d'azote qui lui est finalement néfaste.

\section{Principaux métabolites marqués}

Les analyses ont été effectuées à partir des plantes âgées de 25j.

En ce qui concerne les métabolites solubles (tableau II), les échantillons nitrate se caractérisent par un marquage plus élevé de la fraction anionique, les acides malique et glycérique étant les composés les plus radioactifs; le glycérate pourrait provenir de la déphosphorylation du phosphoglycérate, aucun inhibiteur des phosphatases n'ayant été apporté dans le milieu d'extraction. La biosynthèse d'acides organiques chez les végétaux cultivés sur nitrate a été signalée par de nombreux auteurs : elle a pour but de stabiliser le $\mathrm{pH}$ intracellulaire perturbé par la réduction des ions $\mathrm{NO}_{3}^{-}$(Kirkby et Mengel, 1967; Raven et Smith, 1976; Salsac et al, 1987) et la production d'acide malique par carboxylation du phosphoénolpyruvate jouerait un rôle essentiel (Davies, 1979).

La formation et le stockage d'importantes quantités de molécules azotées organiques, correspondant à un phénomène de détoxification, est également une réponse classique à une alimentation ammoniacale. II s'agit le plus souvent des amides, glutamine et asparagine, ou d'acides aminés basiques tels que l'arginine (Givan, 1979). L'examen du tableaull montre effectivement, chez les plantes «ammonium», une incorporation plus élevée de radiocarbone dans les acides aminés et amides libres $\left(44,2 \%\right.$ du ${ }^{14} \mathrm{C}$ assimilé contre $32,5 \%$ dans les plantes «nitrate»). Cependant, si les radioactivités du glutamate et de la glutamine sont très légèrement supérieures, les marquages de la glycine, et surtout de la sérine, présentent les augmentations les plus nettes. À la suite de la fixation de ${ }^{14} \mathrm{CO}_{2}$ par des végétaux alimentés en azote ammoniacal, un accroissement de la radioactivité de la sérine a aussi été observé chez la luzerne (Platt et al, 1977) et le blé (Walker et al, 1984); en revanche, une constatation inverse a été effectuée chez l'épinard (Larsen et al, 1981; Lawyer et al, 1981). Ces données nous ont conduits à déterminer l'importance quantitative de la sérine dans la fraction azotée so-
Tableau II. Répartition de la radioactivité dans les principaux métabolites solubles. Plantes récoltées au $25^{\mathrm{e}} \mathrm{j}$ de culture; résultats en $\%$ de la radioactivité totale fixée; moyenne de 3 répétitions.

\begin{tabular}{lrr}
\hline \multirow{2}{*}{ Métabolite considéré } & \multicolumn{2}{c}{ Source d'azote } \\
& Nitrate & Ammonium \\
\hline Acides aminés et & & \\
amides libres & 32,5 & $\mathbf{4 4 , 2}$ \\
Glycine & 1,3 & 3,3 \\
Sérine & 11,8 & 29,8 \\
$\alpha$-Alanine & 10,5 & 6,5 \\
Aspartate & 6,9 & 0,8 \\
Glutamate & 1,4 & 2,3 \\
Glutamine & 0,4 & 1,1 \\
Acides organiques & 18,6 & $\mathbf{7 , 4}$ \\
Glycolate & 0,2 & 0,3 \\
Phosphoglycérate & 0,1 & 0,4 \\
Glycérate & 6,9 & 3,0 \\
Succinate & 0,2 & 0,2 \\
Fumarate & 0,1 & 0,1 \\
Malate & 8,9 & 2,3 \\
Citrate & 1,4 & 0,7 \\
Malonate & 0,4 & 0,2 \\
Composés glucidiques & $\mathbf{1 9 , 8}$ & $\mathbf{2 2 , 8}$ \\
Oses-phosphate & 3,6 & 3,4 \\
Glucose & 3,7 & 3,7 \\
Fructose & 1,9 & 1,3 \\
Saccharose & 9,2 & 12,8 \\
Maltose & 1,2 & 1,4 \\
\hline
\end{tabular}

luble des feuilles de soja (tableau III). On note que la sérine est, après l'arginine, l'acide aminé dont les teneurs augmentent le plus dans les plantes cultivées sur $\mathrm{NH}_{4}^{+}$; cependant l'accumulation d'asparagine constitue le phénomène essentiel. Le fait que l'asparagine ne figure pas parmi les premières molécules marquées après assimilation de ${ }^{14} \mathrm{CO}_{2}$ (tableau II) pourrait signifier que ce composé est synthétisé essentiellement dans les racines, puis transporté dans les feuilles où il est stocké.

La nutrition ammoniacale se traduit enfin (tableau II) par une radioactivité légèrement supérieure de la fraction glucidique, dans laquelle le saccharose occupe une place prépondérante.

La fraction insoluble renferme $29,1 \%$ du radiocarbone incorporé dans les échantillons "nitrate", contre $25,6 \%$ dans les échantillons «ammonium».

Après hydrolyse acide d'une partie aliquote du culot et traitement de l'hydrolysat sur résine cationique, la radioactivité de l'éluat correspond au ${ }^{14} \mathrm{C}$ incorporé dans les pro- 
Tableau III. Teneurs en acides aminés et amides libres des limbes de feuilles de soja, en fonction de la composition du milieu de culture. Plantes âgées de $25 \mathrm{j}$ après semis; résultats en $\mu \mathrm{mol} \cdot \mathrm{g}^{-1}$ de matière sèche; moyenne de 3 répétitions.

\begin{tabular}{lcr}
\hline \multicolumn{1}{c}{ Acide aminé } & \multicolumn{2}{c}{ Source d'azote } \\
& Nitrate & Ammonium \\
\hline Glycine & 1,5 & 1,1 \\
Sérine & 6,4 & 23,0 \\
Cystéine & 3,0 & 5,5 \\
Alanine & 4,0 & 14,0 \\
Valine & 1,8 & 5,6 \\
Leucine & 2,2 & 6,3 \\
Aspartate & 17,1 & 5,3 \\
Asparagine & 14,2 & 202,2 \\
Thréonine & 2,7 & 4,3 \\
Isoleucine & 1,4 & 4,6 \\
Lysine & 2,3 & 5,2 \\
Méthionine & 1,5 & 1,4 \\
Glutamate & 9,0 & 17,9 \\
Glutamine & 2,4 & 9,7 \\
Ornithine & 0,6 & 1,5 \\
Arginine & 4,6 & 44,6 \\
Proline & 1,4 & 2,9 \\
$\gamma$-Aminobutyrate & 15,5 & 23,6 \\
Tyrosine & 1,4 & 1,5 \\
Phénylalanine & 1,8 & 4,8 \\
& & \\
Total & 94,8 & 385,0 \\
\hline & &
\end{tabular}

téines. La soustraction de cette valeur au marquage total du culot (déterminé après combustion à l'oxidizer) permet de connaître la radioactivité des constituants insolubles non protéiques, en quasi-totalité de nature glucidique.

Quel que soit l'échantillon analysé, le radiocarbone se répartit en quantités égales dans chacune des 2 fractions. L'aspartate, le glutamate, l'alanine et le glycocolle constituent les principaux amino-acides marqués identifiés, l'amidon étant responsable du marquage de la fraction glucidique.

La diminution de la radioactivité du culot insoluble pourrait donc s'interpréter comme une baisse à la fois de la protéogénèse et de la biosynthèse de l'amidon en réponse à une nutrition ammoniacale. Le premier de ces phénomènes a déjà été mis en évidence au cours d'une étude du catabolisme du glucose ${ }^{14} \mathrm{C}$ (Kpodar, 1987). En ce qui concerne l'amidon, l'étude des teneurs foliaires en composés glucidiques mesurées au début et à la fin de l'héméropériode (tableau IV) apporte des ren- seignements complémentaires. Comparativement aux échantillons «nitrate», on constate en effet que les plantes «ammonium» renferment des quantités supérieures d'amidon en début de journée, l'inverse étant observé à la fin de la phase d'éclairement. Ces résultats plaideraient donc en faveur d'un ralentissement de la dégradation nocturne de l'amidon et de son éventuelle stagnation dans le chloroplaste, mais aussi d'une diminution de sa biosynthèse durant la période d'assimilation photosynthétique. Le carbone fixé est alors utilisé préférentiellement pour l'élaboration de sucres solubles, en grande partie du saccharose, qui s'accumulent dans les feuilles des végétaux cultivés sur $\mathrm{NH}_{4}^{+}$. On note parallèlement une réduction du flux de migration des photosynthétats (Latché, résultats non publiés) vers les autres organes de la plante.

\section{Évolution de quelques paramètres photosynthétiques au cours de la culture}

Les résultats sont regroupés dans le tableau V.

\section{Résistance stomatique}

Les valeurs correspondant aux plantes «ammonium" sont largement supérieures. Cet accroissement se manifeste dès le $20^{\mathrm{e}} \mathrm{j}$ de culture, soit après $5 \mathrm{j}$ de nutrition ammoniacale, et il s'accentue par la suite: par rapport aux végétaux "nitrate", la résistance stomatique est multipliée par environ $1,5,4,6$ et 13,6 respectivement aux $20^{\mathrm{e}}, 25^{\mathrm{e}}$ et $30^{\mathrm{e}} \mathrm{j}$.

\section{Richesse en chlorophylles}

L'apparition de chlorose constitue un des effets courants d'une nutrition ammoniacale. Jusqu'au $25^{\mathrm{e}}$ j de culture, les différences entre soja «nitrate» et «ammonium» sont cependant assez faibles et elles proviennent sans doute d'un défaut de biosynthèse des pigments chez les plantes alimentées en $\mathrm{NH}^{+}{ }_{4}$; on note par la suite une baisse marquée des teneurs en chlorophylles, traduisant l'installation d'un processus de dégradation particulièrement intense à partir du $30^{\mathrm{e}} \mathrm{j}$. 
Tableau IV. Teneurs des limbes foliaires en amidon et en composés glucidiques solubles en fonction de la source azotée. Plantes âgées de $25 \mathrm{j}$ après semis; résultats en $\mathrm{mg}$ d'équivalents glucose. $\mathrm{g}^{-1}$ de matière sèche; moyenne de 4 échantillons; $D$ et $F$ désignent respectivement le début et la fin de l'héméropériode.

\begin{tabular}{lcccc}
\hline Glucide considéré & \multicolumn{2}{c}{ Plantes «nitrate» } & $F$ & \multicolumn{2}{c}{ Plantes «ammonium» } \\
& $D$ & $61,1 \pm 2,9$ & $D$ & $F$ \\
\hline Amidon & $41,3 \pm 2,8$ & $55,1 \pm 2,6$ & $53,4 \pm 2,1$ \\
Glucides solubles & $20,5 \pm 1,9$ & $25,0 \pm 1,8$ & $67,3 \pm 2,1$ & $72,0 \pm 3,2$ \\
\hline
\end{tabular}

Tableau V. Effets de l'alimentation ammoniacale sur la résistance stomatique $\left(\mathrm{s} \cdot \mathrm{m}^{-1}\right)$, les teneurs en chlorophylle, en protéines foliaires solubles et en Rubisco ( $\mathrm{mg} \cdot \mathrm{g}^{-1}$ de MF), les activités carboxylasiques de la Rubisco (activités totales mesurées après préactivation de l'enzyme; ${ }^{a}$ : nKat. $\mathrm{g}^{-1} \cdot \mathrm{MF}$; ${ }^{\mathrm{b}}$ : $\mathrm{nKat} \cdot \mathrm{mg}^{-1} \mathrm{de}$ Rubisco) et son degré d'activation (\%). Les résultats correspondent à la moyenne de 3-5 mesures. ND: non déterminé.

\begin{tabular}{|c|c|c|c|c|c|c|c|c|}
\hline \multirow[b]{2}{*}{$\begin{array}{l}\text { Jours après } \\
\text { semis }\end{array}$} & \multicolumn{4}{|c|}{ Plantes «nitrate» } & \multicolumn{4}{|c|}{ Plantes «ammonium» } \\
\hline & 20 & 25 & 30 & 35 & 20 & 25 & 30 & 35 \\
\hline $\begin{array}{l}\text { Résistance } \\
\text { stomatique }\end{array}$ & $910 \pm 46$ & $570 \pm 36$ & $340 \pm 28$ & ND & $1400 \pm 60$ & $2600 \pm 72$ & $4640 \pm 83$ & ND \\
\hline Chlorophylles & $2,5 \pm 0,2$ & $2,6 \pm 0,2$ & $3,0 \pm 0,3$ & $3,3 \pm 0,3$ & $2,3 \pm 0,2$ & $2,2 \pm 0,2$ & $1,9 \pm 0,1$ & $1,2 \pm 0,1$ \\
\hline $\begin{array}{l}\text { Protéines } \\
\text { solubles }\end{array}$ & $17,4 \pm 0,8$ & $15,0 \pm 0,8$ & $21,1 \pm 1,1$ & $27,8 \pm 1,3$ & $17,6 \pm 0,9$ & $15,5 \pm 0,8$ & $16,0 \pm 0,9$ & $16,7 \pm 0,8$ \\
\hline Rubisco & $8,8 \pm 0,5$ & $11,6 \pm 0,6$ & $18,3 \pm 0,7$ & $23,0 \pm 0,8$ & $8,2 \pm 0,5$ & $8,2 \pm 0,5$ & $8,2 \pm 0,6$ & $8,8 \pm 0,5$ \\
\hline $\begin{array}{l}\text { Activité } \\
\text { carboxylasique }{ }^{a}\end{array}$ & $55 \pm 3,9$ & $95 \pm 5,1$ & $94 \pm 5,0$ & $80 \pm 4,1$ & $62 \pm 3,4$ & $75 \pm 3,6$ & $67 \pm 3,1$ & $43 \pm 4,4$ \\
\hline $\begin{array}{l}\text { Activité } \\
\text { carboxylasique }\end{array}$ & $6,3 \pm 0,4$ & $8,2 \pm 0,5$ & $5,1 \pm 0,4$ & $3,5 \pm 0,4$ & $7,6 \pm 0,6$ & $9,1 \pm 0,7$ & $8,2 \pm 0,7$ & $4,9 \pm 0,5$ \\
\hline Degré d'activation & $78 \pm 4$ & $80 \pm 5$ & $84 \pm 4$ & $72 \pm 4$ & $80 \pm 4$ & $79 \pm 3$ & $80 \pm 4$ & $70 \pm 3$ \\
\hline
\end{tabular}

\section{Évolution des teneurs en protéines solu- bles et en Rubisco}

Chez les plantes de type photosynthétique $\mathrm{C}_{3}$, la Rubisco représente $25-60 \%$ des protéines foliaires extraites en milieu aqueux ( $\mathrm{Ku}$ et al, 1979; Wittenbach et al, 1980; Peoples et al, $1983)$ et des valeurs plus élevées (65-80\%) ont même été reportées (Huffaker et Miller, 1978) notamment chez le soja (Grima-Pettenati, 1985). En alimentation nitrique, les concentrations en Rubisco, encore faibles au premier stade d'étude, augmentent au fur et à mesure que la feuille atteint l'état adulte (tableau V). II en est de même de la part qu'occupe l'enzyme parmi les protéines solubles foliaires, soit environ $51 \%, 77 \%$ et $87 \%$ respectivement aux $20^{\mathrm{e}}, 25^{\mathrm{e}}$ et $30^{\mathrm{e}}$ j de culture; ces dernières valeurs sont particulièrement fortes et leur augmentation au cours du développement de la feuille indique que la biosynthèse de la Rubisco s'effectue avec une intensité nettement plus grande que celle des autres protéines. Toutefois, au $35^{\mathrm{e}} \mathrm{j}$ de culture, le pourcentage correspondant à la Rubisco $(82,7 \%)$ amorce une légère baisse. Elle peut être le signe d'un début de sénescence du limbe qui se traduit alors par une dégradation préférentielle de la Rubisco comparativement aux autres macromolécules azotées (Peterson et Huffaker, 1975; Wittenbach et al, 1980; Laurière, 1983; Grima-Pettenati, 1985).

Par rapport aux échantillons «nitrate», la richesse en protéines foliaires du soja cultivé sur ammonium est inférieure dans les 2 derniers stades analysés; ceci confirme l'hypothèse d'une diminution de la protéogénèse évoquée précédemment. Les teneurs en Ru- 
bisco, également plus faibles, restent à peu près constantes en valeur absolue en fonction de l'âge de la feuille; il en est de même de la part que représente l'enzyme dans la fraction protéique (47-53\%). Lorsque l'azote est fourni sous forme ammoniacale, on assisterait donc à un ralentissement précoce, sinon à un arrêt, de la biosynthèse de la Rubisco.

\section{Activités carboxylasiques de la Rubisco}

L'expression des résultats par rapport au g de matière fraîche fait apparaître, dans les plantes témoins cultivées sur azote nitrique, une évolution analogue à celle constatée au cours d'un travail antérieur (Latché et al, 1986). La feuille encore en cours de croissance présente les activités les plus faibles; un maximum, correspondant à l'état adulte, est ensuite observé et une baisse s'amorce dans les échantillons plus âgés. L'apport d'azote sous forme d'ions $\mathrm{NH}_{4}^{+}$entraîne d'abord une légère augmentation de l'activité carboxylasique de la Rubisco (+12\%). Une diminution se manifeste par la suite si la nutrition ammoniacale se prolonge; elle atteint $21 \%, 29 \%$ et $46 \%$ respectivement aux $25^{\mathrm{e}}, 30^{\mathrm{e}}$ et $35^{\mathrm{e}} \mathrm{j}$ de culture.

De l'examen du tableau $V$ il ressort également que l'activité carboxylasique spécifique (nKat. $\mathrm{mg}^{-1}$ de protéine enzymatique dosée par immunoélectrophorèse) diffère en fonction de l'âge de la feuille. Les valeurs maximales sont obtenues à partir des organes récoltés $25 \mathrm{j}$ après semis et ce sont aux feuilles les plus âgées $\left(35^{\mathrm{e}} \mathrm{j}\right.$ de culture) que correspondent les chiffres les plus faibles. Cette évolution est identique dans les 2 types d'échantillons, mais les activités spécifiques sont toujours un peu plus élevées dans les plantes «ammonium». Selon Hall et al (1981) tous les sites catalytiques de la Rubisco ne sont pas fonctionnels et, à l'intérieur d'une population de molécules enzymatiques, certaines sont inactives alors que d'autres exercent leur fonction de catalyse au maximum de leurs capacités. Ceci expliquerait que l'activité carboxylasique spécifique varie, si on admet que les possibilités d'activation de l'enzyme, et donc les proportions de molécules actives et inactives, puissent différer selon l'âge des chloroplastes par exemple. À l'appui de cette hypothèse, on constate (tableau V) que le degré d'activation de l'enzyme, déterminé à par- tir de la mesure des activités totale et initiale, diminue légèrement dans les feuilles âgées $\left(80-84 \%\right.$ aux $25^{\mathrm{e}}$ et $30^{\mathrm{e}} \mathrm{j}$ de culture, contre $72 \%$ au $35^{\mathrm{e}} \mathrm{j}$ ). En revanche, la nature de la source azotée, nitrate ou ammonium, n'a pas d'influence significative sur le degré d'activation.

\section{DISCUSSION ET CONCLUSION}

Le tableau VI regroupe les valeurs du rapport entre les principales données numériques acquises à partir des plants de soja cultivés sur ammonium et celles obtenues à partir des échantillons «nitrate» correspondants. Plus ce rapport s'écarte de l'unité, plus les modifications enregistrées en nutrition ammoniacale sont intenses.

On constate d'abord l'existence d'une période d'apparente tolérance aux ions $\mathrm{NH}_{4}^{+}$d'au moins $5 \mathrm{j}$, durant laquelle le végétal ne réagit que très faiblement, et même parfois de façon positive. Ainsi, l'activité ribulose 1,5 bisphosphate carboxylase s'avère légèrement supérieure dans les plantes «ammonium", résultat également reporté chez l'épinard (De Benedetti et al, 1976), le haricot (Marques et al, 1983) et le blé (Hall et al, 1984).

Les causes de la baisse de photosynthèse nette observée par la suite sont multiples. La plus précoce, et également la plus intense, paraît être l'augmentation de la résistance stomatique. La fermeture des stomates en relation avec la nutrition azotée n'avait surtout été signalée jusqu'ici que dans des situations de carence, à la fois chez le soja (Grima-Pettenati et al, 1986) et chez d'autres végétaux tels que le tournesol (Goldbach et al, 1975) et le cotonnier (Radin et Ackerson, 1981); dans ces

Tableau VI. Principaux effets de l'alimentation ammoniacale, illustrés par le rapport des résultats échantillons ammonium

échantillons nitrate

\begin{tabular}{lccccc}
\hline $\begin{array}{c}\text { Jours de culture sur } \\
\text { milieu ammonium }\end{array}$ & 5 & 10 & 15 & 20 \\
\hline Photosynthèse nette & 0,98 & 0,65 & 0,87 & 0,72 \\
Résistance stomatique & 1,54 & 4,56 & 13,64 & - \\
Prot solubles foliaires & 1,01 & 1,03 & 0,76 & 0,60 \\
Teneurs en Rubisco & 0,93 & 0,71 & 0,45 & 0,38 \\
Teneurs en chlorophylles & 0,92 & 0,85 & 0,63 & 0,36 \\
Activ carboxyl Rubisco & 1,13 & 0,79 & 0,71 & 0,54 \\
\hline
\end{tabular}


2 derniers cas, elle a été corrélée avec une production d'acide abscissique.

On note ensuite (tableau VI), outre la baisse des teneurs en chlorophylle, une diminution de l'activité carboxylasique rapportée à la masse de matière fraîche, ainsi qu'une déficience en protéine enzymatique. Cette dernière semble surtout imputable à une inhibition de la biosynthèse de la Rubisco, qui se manifesterait de façon sélective puisque parallèlement les teneurs globales en protéines foliaires sont moins affectées. Ce phénomène mériterait toutefois confirmation, par exemple par marquage de la protéogénèse in vivo. De même, les variations de l'activité carboxylasique spécifique, et notamment du degré d'activation de la Rubisco en fonction de l'âge de la feuille et de l'alimentation en azote, doivent faire l'objet d'un prochain travail.

Par ailleurs la répartition du carbone assimilé varie également. Deux faits méritent d'être soulignés. En premier lieu, à la suite de l'incorporation de ${ }^{14} \mathrm{CO}_{2}$, les plantes «ammonium» se caractérisent par un marquage beaucoup plus élevé de la sérine et l'importance de ce composé dans la fraction acides aminés libres est également supérieure. Ceci suggère que le stockage de la sérine participe à la désintoxication des cellules végétales en réponse à de trop fortes teneurs en ions $\mathrm{NH}_{4}^{+}$; ce résultat a été assez peu souvent mentionné, bien que déjà observé chez le sarrasin (Kpodar et al, 1978), le blé (Walker et al, 1984) et le haricot (Chaillou et al, 1986). En second lieu, la formation de saccharose semble s'effectuer de façon préférentielle en nutrition ammoniacale, au détriment de la biosynthèse d'amidon. De même que les acides aminés et amides libres (Salsac et al, 1987), le saccharose joue sans doute un rôle important dans le maintien de la pression osmotique du suc vacuolaire des cellules foliaires, rôle tenu essentiellement par les acides organiques dans les plantes cultivées sur nitrate.

On remarque enfin que le soja peut réagir de façon similaire en réponse à une alimentation ammoniacale d'une part, à une privation d'azote d'autre part (Grima-Pettenati, 1985). Selon Tolley-Henry et Raper (1986), l'acidification de la solution nutritive, consécutive au développement des végétaux sur ammonium, provoque un ralentissement de l'absorption des ions $\mathrm{NH}_{4}^{+}$pouvant conduire, s'il se pro- longe, à une situation de carence en azote. Toutefois ce phénomène n'est pas observé si le $\mathrm{pH}$ du milieu se situe entre 5 et 6 et il n'apparaît nettement qu'à $\mathrm{pH} 4,5$ (Raper et al, 1991). Dans les conditions de culture adoptées au cours du présent travail, une acidification aussi importante de la solution n'a jamais été caractérisée, le $\mathrm{pH}$ restant compris entre 5,1 et 5,6 pour les premiers stades de récolte et entre 4,9 et 5,6 lorsque les plantes sont plus âgées. Il semble donc que, même si une baisse de l'absorption racinaire des ions $\mathrm{NH}_{4}^{+}$se manifestait, elle ne serait pas suffisante pour provoquer une carence azotée, et que les perturbations enregistrées dans le métabolisme photosynthétique du soja soient essentiellement la conséquence de l'apport d'azote sous forme ammoniacale. Différentes étapes sont touchées, telles que l'approvisionnement en anhydride carbonique du parenchyme foliaire, la capture de l'énergie lumineuse par le chloroplaste, le déroulement de la réaction de carboxylation proprement dite; l'exportation du carbone fixé, des feuilles vers les organes "puits", est également ralentie. D'un point de vue plus général, ces résultats illustrent la multiplicité des interactions entre nutrition carbonée et nutrition azotée.

\section{RÉFÉRENCES}

Archhireddy NR, Vann DR, Fletcher JS, Beevers $L$ (1983) The influence of methionine sulfoximine on phtosynthesis and nitrogen metabolism in excised pepper (Capsicum annuum $L$ ) leaves. Plant Sci Lett 32, 73-78

Arnon DI (1949) Copper enzymes in isolated chloroplasts. Polyphenol oxidase in Beta vulgaris. Plant Physiol 24, 1-15

Bensadoun A, Weinstein D (1976) Assay for proteins in the presence of interfering materials. Anal Biochem 70, 241-250

Bernardo LM, Clark RB, Maranville JW (1984) Nitrate/ammonium ratio effects on nutrient solution $\mathrm{pH}$, dry matter yield, and nitrogen uptake of Sorghum. J Plant Nutr 7, 1389-1400

Bruinsma $J$ (1961) A comment on the spectrophotometric determination of chlorophyll. Biochim Biophys Acta 52, 575-578

Chaillou S, Morot-Gaudry JF, Lesaint C, Salsac L, Jolivet $E$ (1986) Compared effects of $\mathrm{NO}_{3}$ and $\mathrm{NH}_{4}$ on growth and metabolism of French bean. Physiol Vég 24, 679-687 
Courtiade B (1983) La ribulose bisphosphate carboxylase-oxygénase du tournesol: dosage et évolution ontogénique. Thèse Doct $3^{\mathrm{e}}$ Cycle, Univ Paul Sabatier, Toulouse

Cruiziat P, Thomas DA, Bodet C (1979) Comparaison entre mesures locales et mesures giobales de la résistance stomatique des feuilles de tournesol. CEcol Plant 14, 447-459

Davies DD (1979) The central role of phosphoenol pyruvate in plant metabolism. Ann Rev Plant Physiol 30, 131-158

De Benedetti E, Forti G, Garlaschi FM, Rosa L (1976) On the mechanism of ammonium stimulation of photosynthesis in isolated chloroplasts. Plant Sci Lett 7, 85-90

Givan CV (1979) Metabolic detoxification of ammonia in tissues of higher plants. Phytochem $18,375-382$

Goldbach E, Goldbach H, Wagner H, Michael G (1975) Influence of $\mathrm{N}$-deficiency on the abscissic acid content of sunflower plants. Physiol Plant 34, 138-140

Grima-Pettenati J (1985) Effets d'une privation d'azote sur la nutrition carbonée et la dégradation des protéines foliaires chez le soja. Thèse Doct. $3^{e}$ cycle, Univ Paul Sabatier, Toulouse

Grima-Pettenati J, Piquemal M, Latché JC (1986). Effets d'une privation d'azote sur le métabolisme photosynthétique chez le soja. Physiol Vég 24, 569-580

Grumbles RM (1987) The effect of glutamate synthase deficiency and ammonia on Arabidopsis metabolism. J Plant Physiol 130, 363-371

Guitton $Y$ (1964) Métabolisme de l'arginine dans les premiers stades de développement de Pinus pinea L. Physiol Vég 2, 95-156

Hall NP, McCurry SD, Tolbert NE (1981) Storage and maintaining activity of $\mathrm{RuBPC} / \mathrm{Ox}$. Plant Physiol 67, 1220-1223

Hall NP, Reggiani R, Franklin J, Keys AJ, Léa PJ (1984) An investigation into the interaction between nitrogen nutrition, photosynthesis and photorespiration. Photosynth Res 5 361-369

Huffaker RC, Miller BL (1978) Reutilization of ribulose bisphosphate carboxylase. In: Photosynthetic Carbon Assimilation (HW Siegelman, G Hind eds) Plenum Press, New York, 139-152

Kirkby EA, Mengel K (1967) lonic balance in different tissues of the tomato plant in relation to nitrate, urea or ammonium nutrition. Plant Physiol 42, 6-14

Kpodar MP (1987) Incidence d'une alimentation azotée ammoniacale sur le métabolisme carboné du maïs, du sarrasin, du soja et du tournesol. Thèse Doct Etat, Univ Paul Sabatier, Toulouse

Kpodar MP, Piquemal M, Calmès J, Latché JC (1978) Relations entre nutrition azotée et méta- bolisme photorespiratoire chez une plante à oxalate, Fagopyrum esculentum M. Physiol Vég $16,117-130$

Ku MFB, Schmitt MR, Edwards GE (1979) Quantitative determination of RuBP carboxylase-oxygenase protein in leaves of several $\mathrm{C}_{3}$ and $\mathrm{C}_{4}$ plants. J Exp Bot 30, 89-98

Larsen PO, Cornwell KL, Gee SL, Bassham JA (1981) Amino acid synthesis in photosynthesizing spinach cell. Plant Physiol 68, 292-299

Latché JC, Ranty B, Calmès J, Abravanel G, Cavalié $G$ (1983) Action de l'hydroxybutynoate de butyle sur la photosynthèse et le métabolisme photorespiratoire. Physiol Vég 21, 435-445

Latché JC, Bailly-Fenech G, Grima-Pettenati J, Cavalié $G$ (1986) Étude comparative du métabolisme photosynthétique des feuilles et des gousses de soja, Glycine max. Can J Bot 64 , 1542-1548

Laurell CB (1966) Quantitative estimation of proteins by electrophoresis in agarose gel containing antibodies. Anal Biochem 15, 45-52

Laurière C (1983) Évolution et aspects de la régulation de plusieurs enzymes en relation avec la sénescence foliaire du blé. Thèse Doct Etat, Univ Paris VI

Lawyer AL, Cornwell KL, Larsen PO, Bassham JA (1981) Effects on the regulation of photosynthetic carbon metabolism by ammonia in spinach mesophyll cells. Plant Physiol 68, 1231-1236

Lowry $\mathrm{OH}$, Rosebrough NJ, Farr AL, Randall RJ (1951) Protein measurement with the Folin phenol reagent. J Biol Chem 193, 265-275

Mac Leod KC, Ormrod DC (1985) Responses of white bean to ammonium or nitrate nutrition at three temperatures. Can J Plant Sci 65, 201-205

Magalhaes JR, Wilcox GE (1984) Ammonium toxicity development in tomato plants relative to $\mathrm{ni}$ trogen form and light intensity. J Plant Nutr 7 , 1477-1496

Marques JA, Oberholzer MJ, Erisman KH (1983) Effect of different inorganic nitrogen sources on photosynthetic carbon metabolism in primary leaves of non-nodulated Phaseolus vulgaris $L$. Plant Physiol 71, 555-561

Mondal MH, Brenner MI (1978) Effects of sink removal on photosynthesis and senescence in leaves of soybean (Glycine max L) plants. Plant Physiol 61, 394-397

Peoples MB, Pate JS, Atkins CA (1983) Mobilization of nitrogen in fruiting plants of a cultivar of cowpea. J Exp Bot 42, 563-578

Peterson LW, Huffaker RC (1975) Loss of ribulose 1,5 diphosphate carboxylase and increasing in proteolytic activity during senescence of detached primary barley leaves. Plant Physiol 55, 1009-1015 
Platt SG, Plaut Z, Bassham JA (1977) Ammonia regulation of carbon metabolism in phtosynthesizing leaf discs. Plant Physiol 60, 739-742

Radin JW, Ackerson RC (1981) Water relations of cotton plants under nitrogen deficiency. III. Stomatal conductance, photosynthesis, and abscissic acid accumulation during drought. Plant Physiol 67, 115-119

Raghavendra AS, Das VS (1977) Purification and properties of PEPCase and RuBPCase $\mathrm{C}_{4}$ and $\mathrm{C}_{3}$. Z Pflanzenphysiol 82, 315-321

Ranty B, Cavalié G (1982) Purification and properties of ribulose 1,5-bisphosphate carboxylase from sunflower leaves. Planta 155, 388-391

Raper CD, Vessey JK, Henry LT, Chaillou S (1991) Cyclic variations in nitrogen uptake rate of soybean plants: effects of $\mathrm{pH}$ and mixed nitrogen sources. Plant Physiol Biochem 29, 205-212

Raven JH, Smith FA (1976) Nitrogen assimilation and transport in vascular land plants in relation to intracellular pH regulation. New Phytol 76 415-431

Rehfeld DW, Jensen RG (1973) Metabolism of separated leaf cells. Plant Physiol 52, 17-22

Rufty TW, Huber SC (1983) Changes in starch formation and activities of sucrose phosphate synthetase and cytoplasmic fructose 1,6-bisphosphatase in response to source-sink alterations. Plant Physiol 72, 474-480

Salsac L, Chaillou S, Morot-Gaudry JF, Lesaint C, Jolivet $E$ (1987) Nitrate nutrition or ammonium nutrition in plants (a review). Plant Physiol Biochem 25, 805-812

Secor J, Ford DM, Shibles R (1982) Ontogenic changes in ribulose-1,5-bisphosphate carboxylase-oxygenase activity in soybean leaves. Plant Sci Lett 27, 147-154
Sharma SN, Sirohi GS (1987) The effect of ammonium and nitrate on $\mathrm{CO}_{2}$ assimilation, RuBP and PEP carboxylase activity and dry matter production in wheat. Photosynth Res 12, 265272

Shelp BJ (1987) Plant characteristics and nutrient composition and mobility of broccoli (Brassica oleracea var italica) supplied with $\mathrm{NH}_{4}^{+}, \mathrm{NO}_{3}^{-}$, or $\mathrm{NH}_{4} \mathrm{NO}_{3}$. J Exp Bot 38, 1603-1618.

Shields R, Burnett S (1960) Determination of sugars. Anal Chem 32, 885-888

Tolley-Henry L, Raper CD (1986) Utilization of ammonium as a nitrogen source. Effects of ambient acidity on growth and nitrogen accumulation by soybean. Plant Physiol 82, 54-60

Torisky RS, Servaites JC (1984) Effect of irradiance during growth of Glycine max on photosynthetic capacity and percent activation of ribulose 1,5 bisphosphate carboxylase. Photosynth Res 5, 251-261

Wakiuchi N, Matsumoto N, Takahashi E (1971) Changes of some enzyme activities of cucumber during ammonium toxicity. Physiol Plant 24, 248-253

Walker KA, Keys AJ, Givans CV (1984) Effect of L-methionine sulfoximine on the products of photosynthesis in wheat (Triticum vulgare) leaves. J Exp Bot 35, 1800-1810

Wittenbach VA, Ackerson RC, Giaquinta RT, Hebert RR (1980) Changes in photosynthesis, ribulose bisphosphate carboxylase, proteolytic activity and ultrastructure of soybean leaves during senescence. Crop Sci 20, 225-231

Woo KC, Canvin DT (1980) Effect of ammonia on photosynthetic carbon fixation in isolated spinach leaf cells. Can J Bot 58, 505-510 